\author{
Assistant Professor Muhammad SHERAZ, PhD \\ E-mail: msheraz@iba.edu.pk \\ Department of Mathematical Sciences \\ Institute of Business Administration Karachi, Pakistan \\ Associate Professor Silvia DEDU, PhD \\ E-mail: silvia.dedu@ csie.ase.ro \\ Department of Applied Mathematics \\ The Bucharest University of Economic Studies
}

\title{
BITCOIN CASH: STOCHASTIC MODELS OF FAT-TAIL RETURNS AND RISK MODELING
}

\begin{abstract}
Bitcoin (BTC) is a digital currency that has gained significant attention from researchers. The aim of this paper consists in analyzing some stochastic models of fat-tail returns and risk models. The evidence of fat-tailed returns distribution for the $B C H$ data is investigated, by performing a statistical analysis of Bitcoin Cash (BCH) in the U.S. dollar. By using daily Close, Open, Low, and High returns of BCH data series, the monthly-divided daily returns study describes further properties such as skewness, kurtosis, and correlation analysis. The results obtained prove that variance gamma distribution best fit the close, open and low returns, where high returns follow the generalized hyperbolic distribution. In addition, for the best-fitted fat-tailed returns distributions, several risk measures such as volatility, Value-at-Risk and Expected Shortfall measures are computed, analyzed and compared.
\end{abstract}

Keywords: Cryptocurrency, Fat-tail distributions, Value-at-Risk, Bitcoin Cash, Expected Shortfall.

\section{JEL Classification: C01, C02, C32, C52, C53}

\section{Introduction and Literature Review}

Since 2009, numerous cryptocurrencies have been developed (CoinMarketCap, 2019). In the list of cryptocurrencies Bitcoin is the most popular, representing the highest market cap. Bitcoin Cash $(\mathrm{BCH})$ is a branch of Bitcoin (BTC), as by August 2017 the bitcoin ledger has divided into two assets. Therefore, anyone owning bitcoin was also in possession of the same number of Bitcoin Cash units. BTC \& BCH share the same transaction history. Recently, the research on cryptocurrencies is receiving significant attention. Bitcoin is probably the most debatable and successful among the list of all cryptocurrencies to date. Nowadays Bitcoin is considered as an asset rather than a currency. If we want to understand

DOI: 10.24818/18423264/54.3.20.03 
the market dynamics of cryptocurrency, then it is important to analyze the statistical properties of their returns. At this point, it is vital to investigate whether these returns behave similarly to other assets. Examples of such assets include currency, commodity or a stock. Therefore, it is now important to study the statistical properties of $\mathrm{BCH}$, which represents a branch of BTC.

The random variation in asset prices share some common properties. These common facts among various markets, such as stocks, instruments for example 'derivatives' and time periods, are called stylized facts. Therefore, in general, stock returns and foreign exchange rates exhibit leptokurtosis and stochastic volatility. Rama Cont (2001) investigated facts emerging from the statistical analysis of price variations in various types of financial markets. The stylized statistical properties of a financial asset may include heavy-tail (conditional/unconditional), Normally distributed returns, volatility clustering, autocorrelation and leverage effect. In financial time series prices usually down movements are frequent that of the upward. It validates the presence of asymmetry in aggregate loss and gain.

Any distribution can be characterized by using a number of features such as mean, variance, skewness and kurtosis. The measure of kurtosis is considered to assess whether the data are peaked or flat relative to the Normal distribution. Mandelbrot (1963) provided a fat-tail distribution model in finance. BarndorffNielsen (1977) introduced the generalized hyperbolic distribution (GHD). Lévy stochastic models have been proposed in early 1980s, since when different stylized features of financial assets have been introduced. These models have been extensively used in financial literature, see for example Schoutens (2003). Eberlein and Keller (1995) investigated the application of hyperbolic distributions in finance. Corlu et al. (2015) performed the modelling of exchange rates and their suitable distributions. Linden and Mikael (2001) model stock share returns using the Laplace mixture distribution. Recently, Chu et al. (2015) provided a statistical analysis of log-returns of the exchange rate of BTC. Dyhrberg (2016) modeled and predicted the volatility of BTC, gold and dollar. Chan et al. (2017) have obtained that the generalized hyperbolic distribution gives the best fit for the Bitcoin and LiteCoin and also they have concluded that the normal inverse Gaussian distribution gives the best fit for other cryptocurrencies which include Dash, Monero and Ripple. Bariveara et al. (2017) have performed a comparative analysis of Bitcoin and standard currencies dynamics. Their study focuses on the analysis of returns at different time scales. In Osterrieder and Lorenz (2017), a statistical assessment of Bitcoin and its extreme tail behavior have been performed. In risk management the standard deviation (SD) represents a commonly used risk measure. Value-at-Risk (VaR) is a quantile based risk measure suitable for nonnormal distributions. For heavy-tailed distribution the risk measure has extreme behaviour. A variety of literature on Value-at-Risk (VaR) and Expected Shortfall (ES) can be accessed following Artezner (1997), RiskMetrics Group (1994), Artezner et al. (1999), Jorion (2001) and McNeil et al. (2005). 
The paper is developed as follows. Section 2 is dedicated to a statistical overview of the underlying data and its descriptions. Section 3 examines the statistical properties of the Bitcoin Cash data by fitting a wide range of fat-tail parametric distributions to the data. Section 4 presents our results and discussion on fitted distribution and risk modelling. Section 5 concludes the paper.

\section{Data}

The data used include the daily Open, Close, Low and High prices (in US dollar) for the $\mathrm{BCH}$ time series, available online at http://www.coindesk.com/price. A time series consisting in 235 observations has been considered, from $31^{\text {th }}$ May 2018 to $30^{\text {th }}$ January 2019 . Let $x_{t}, t \in[0, T]$ denote the $\mathrm{BCH}$ prices data. The percentage logarithmic returns of daily prices are given by:

$$
R_{t}=\log \left(\frac{x_{t}}{x_{t-1}}\right) \times 100, t \in[0, T] .
$$

Summary statistics of the underlying log returns of the exchange rates of $\mathrm{BCH}$ (USD) are presented in Tables 1 and 2, respectively. Table 1 displays the summary statistics of $\mathrm{BCH}$ data series from $31^{\text {th }}$ May 2018 to $30^{\text {th }}$ Jan 2019. The results indicate that the lowest daily return of approximately A share of $57 \%$ corresponds to the Low-returns data from Jun-2018 to Jan-2019, while the best day in the data series, approximately $56 \%$, was found. The returns have a small positive skewness which is slightly high for the case of High-returns data. More importantly, quite high kurtosis has found again for the Low-returns. The daily mean of Open-Low and High-Close returns series are the same. The Jarque-Bera (JB) test clearly rejects that data is normally distributed. The Augmented DickeyFuller (ADF) test suggests the small p-values therefore, returns exhibit stationarity. According to Ljung-Box (LB) test only Low-prices data series exhibits independence. The daily mean is at most one-tenth of the daily volatility of Lowreturns. Therefore, it leads to the complexity of the risk measures. The evidence of high fluctuation in $\mathrm{BCH}$ returns volatility is also evident from Table 1.

Table 1. BCH (USD) Basic Statistics

\begin{tabular}{lllll}
\hline Returns & Close & Open & High & Low \\
\hline No Obs. & 235 & 235 & 235 & 235 \\
Min & -36.51 & -37.32 & -35.52 & -56.37 \\
Max & 37.82 & 37.62 & 33.98 & 55.61 \\
Mean & -0.90 & -0.94 & -0.90 & -0.94 \\
Variance & 50.03 & 49.57 & 47.59 & 70.81 \\
Skewness & 0.12 & 0.044 & 0.27 & 0.059 \\
Kurtosis & 9.99 & 10.37 & 9.65 & 20.60 \\
JB & 0.00 & 0.00 & 0.00 & 0.00 \\
LB & 0.0001 & 0.000 & 0.0007 & 0.446 \\
ADF & 0.01 & 0.01 & 0.01 & 0.01 \\
\hline
\end{tabular}

On the other hand, the Low-returns series is highly-peaked and volatile as compared to others, as can be seen in Figure 1.

DOI: 10.24818/18423264/54.3.20.03 


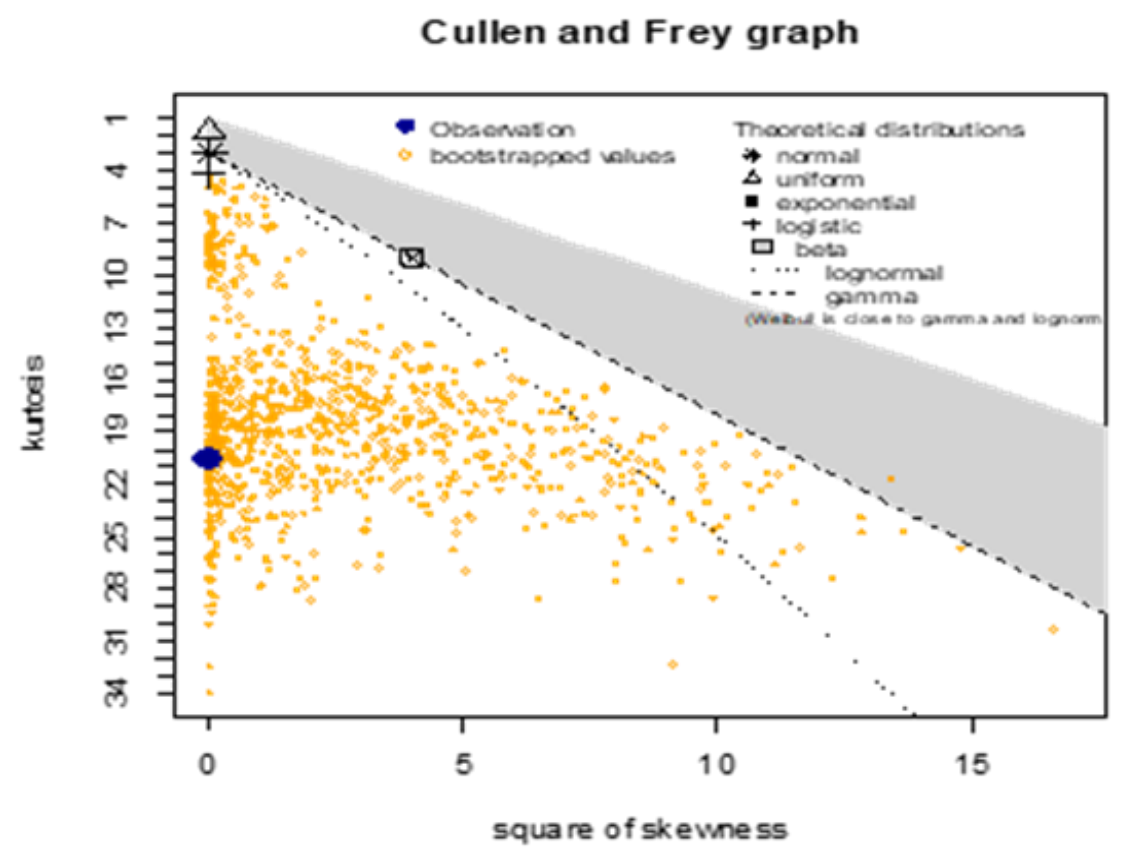

Figure 1. Kurtosis of BCH Low Returns

Further, we consider percentage log-returns of each month of $\mathrm{BCH}$ daily data series. For this purpose, the whole dataset has been divided into months. In the case of month-wise daily data returns we have obtained the unstable daily mean in all four possible states. An increasing volatility pattern has been noticed during the period Nov-2018 to Jan-2019. The monthly divided returns have both negatively and positively skewed distribution while the September to October 2018 returns has higher kurtosis value which leads to fat-tail behavior. During the month of Jan2019, the Low-returns data series exhibit heavier tail as compared to all others. Volatility has been frequently used as the most common measure of market uncertainty. We remark that month-wise daily returns demonstrate volatility clustering property. Table 2 displays monthly wise summary statistics of $\mathrm{BCH}$ data series.

Table 2. Summary Statistics of BCH Monthly Return Series

\begin{tabular}{llllllllll}
\hline Month & No Obs. & Min & Max & Mean & SD & Skew & Kurt & JB & LB \\
\hline Jun & 25 & -19.11 & 8.36 & -1.24 & 6.30 & -0.87 & 3.78 & 0.08 & 0.33 \\
& & -21.32 & 8.29 & -1.22 & 6.93 & -0.95 & 3.88 & 0.05 & 0.74 \\
& & -16.49 & 8.81 & -1.27 & 6.50 & -0.77 & 4.11 & 0.00 & 0.71 \\
& & -19.79 & 8.29 & -1.28 & 6.29 & -1.20 & 5.32 & 0.26 & 0.01 \\
Jul & 29 & -6.78 & 9.60 & 0.22 & 4.50 & 0.54 & 2.44 & 0.40 & 0.43 \\
& & -7.54 & 10.87 & 0.12 & 4.51 & 0.53 & 2.70 & 0.46 & 0.70 \\
\hline
\end{tabular}


Bitcoin Cash: Stochastic Models of Fat-Tail Returns and Risk Modeling

\begin{tabular}{|c|c|c|c|c|c|c|c|c|c|}
\hline & & -6.65 & 9.01 & 0.00 & 3.51 & 0.43 & 3.24 & 0.28 & 0.21 \\
\hline & & -7.16 & 10.50 & -0.10 & 4.06 & 0.68 & 2.83 & 0.66 & 0.83 \\
\hline \multirow[t]{4}{*}{ Aug } & 31 & -10.45 & 13.73 & -0.42 & 5.71 & 0.37 & 3.17 & 0.61 & 0.79 \\
\hline & & -11.63 & 13.46 & -0.68 & 5.72 & 0.38 & 3.35 & 0.52 & 0.82 \\
\hline & & -11.00 & 10.50 & -0.59 & 4.79 & -0.43 & 3.58 & 0.02 & 0.82 \\
\hline & & -10.26 & 16.42 & -0.51 & 5.41 & 0.78 & 4.47 & 0.66 & 0.86 \\
\hline \multirow[t]{4}{*}{ Sep } & 29 & -14.87 & 19.97 & -0.56 & 5.43 & 1.15 & 8.95 & 0.00 & 0.99 \\
\hline & & -15.08 & 19.90 & -0.47 & 5.48 & 1.07 & 8.63 & 0.00 & 0.10 \\
\hline & & -17.11 & 16.94 & -0.49 & 5.13 & 0.02 & 10.69 & 0.00 & 0.99 \\
\hline & & -15.39 & 20.53 & -0.60 & 5.27 & 1.46 & 11.13 & 0.00 & 0.99 \\
\hline \multirow[t]{4}{*}{ Oct } & 31 & -14.59 & 8.53 & -0.53 & 3.72 & -1.31 & 7.84 & 0.00 & 0.83 \\
\hline & & -14.69 & 5.15 & -0.75 & 3.40 & -2.12 & 9.48 & 0.00 & 0.46 \\
\hline & & -14.77 & 5.86 & -0.68 & 3.66 & -1.90 & 9.55 & 0.00 & 0.59 \\
\hline & & -12.13 & 12.83 & -0.50 & 4.21 & 0.68 & 6.60 & 0.00 & 0.32 \\
\hline \multirow[t]{4}{*}{ Nov } & 30 & -23.77 & 16.34 & -2.55 & 8.26 & -0.30 & 3.48 & 0.55 & 0.00 \\
\hline & & -23.11 & 16.30 & -2.43 & 8.12 & -0.35 & 3.50 & 0.49 & 0.00 \\
\hline & & -20.52 & 13.35 & -2.43 & 8.78 & -0.21 & 2.56 & 0.37 & 0.02 \\
\hline & & -20.12 & 19.35 & -2.52 & 9.90 & 0.33 & 3.77 & 0.74 & 0.00 \\
\hline \multirow[t]{4}{*}{ Dec } & 29 & -36.51 & 37.82 & -0.88 & 13.45 & 0.35 & 4.71 & 0.05 & 0.04 \\
\hline & & -37.32 & 37.62 & -0.84 & 13.36 & 0.29 & 4.91 & 0.03 & 0.03 \\
\hline & & -31.19 & 35.34 & -0.85 & 12.30 & 0.45 & 5.31 & 0.23 & 0.05 \\
\hline & & -35.52 & 33.98 & -0.98 & 13.35 & 0.31 & 4.10 & 0.10 & 0.00 \\
\hline \multirow[t]{4}{*}{ Jan } & 30 & -18.06 & 7.67 & -1.24 & 5.18 & -1.03 & 5.08 & 0.00 & 0.78 \\
\hline & & -18.00 & 7.68 & -1.34 & 4.92 & -1.19 & 5.66 & 0.00 & 0.81 \\
\hline & & -56.37 & 55.61 & -1.32 & 15.41 & 0.12 & 14.55 & 0.00 & 0.89 \\
\hline & & -20.17 & 7.48 & 1.14 & 5.02 & -1.66 & 8.09 & 0.00 & 0.82 \\
\hline
\end{tabular}

Figure 2 reveals combined time series plot of daily Close, Open, Low and High returns for both the whole data series and monthly divided series.
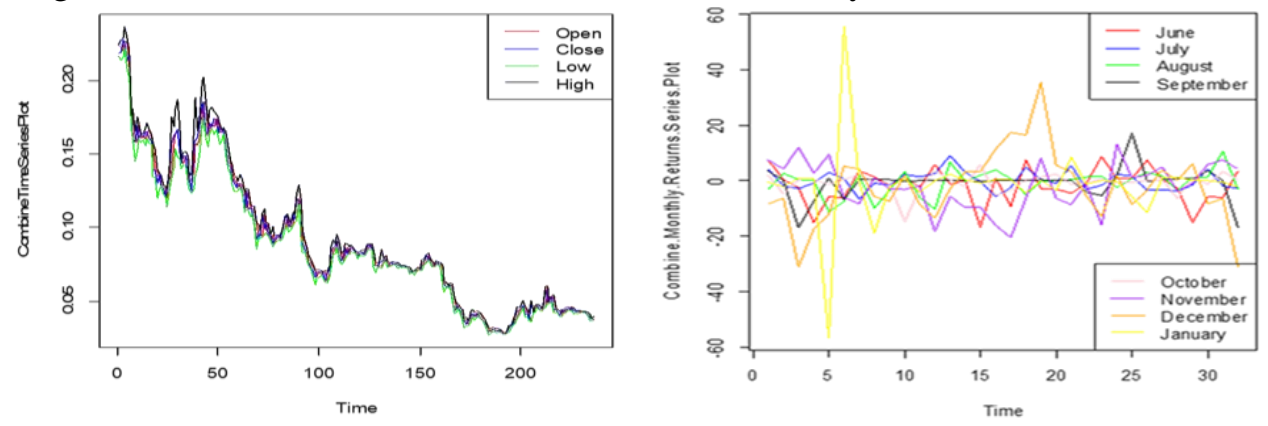

Figure 2. Time Series plot of BCH (USD) returns

The results presented in Table 3 reveal the strength of the dependence between Open-Close-Low-High return series for the whole period. A positive

DOI: 10.24818/18423264/54.3.20.03 
correlation exists in all cases where no evidence of inverse correlation has been found.

Table 3. Correlation matrix of BCH (USD)

\begin{tabular}{lcccc}
\hline & Open & Close & Low & High \\
\hline Open & 1.000 & 0.263 & 0.458 & 0.691 \\
Close & 0.263 & 1.000 & 0.557 & 0.637 \\
Low & 0.458 & 0.557 & 1.000 & 0.415 \\
High & 0.691 & 0.637 & 0.415 & 1.000 \\
\hline
\end{tabular}

The results displayed in Table 4 indicate that month-wise daily returns have a relatively high correlation for the months of Jul-Sep, Sep-Dec, and NovDec-Jan 2019.

Table 4. Correlation analysis of BCH (USD) monthly divided daily closed returns

\begin{tabular}{lllllllll}
\hline & Jun & Jul & Aug & Sep & Oct & Nov & Dec & Jan \\
\hline Jun & 1.00 & 0.03 & -0.14 & 0.04 & -0.11 & -0.05 & -0.11 & -0.09 \\
Jul & 0.03 & 1.00 & -0.18 & 0.29 & 0.01 & 0.06 & 0.04 & -0.02 \\
Aug & -0.14 & -0.18 & 1.00 & 0.05 & -0.06 & -0.00 & 0.13 & 0.18 \\
Sep & 0.04 & 0.29 & 0.05 & 1.00 & -0.08 & -0.07 & 0.42 & -0.20 \\
Oct & -0.11 & 0.01 & -0.06 & -0.08 & 1.00 & 0.06 & -0.09 & 0.02 \\
Nov & -0.05 & 0.06 & -0.00 & -0.07 & 0.06 & 1.00 & -0.28 & -0.25 \\
Dec & -0.11 & 0.04 & 0.13 & 0.42 & -0.09 & -0.28 & 1.00 & 0.18 \\
Jan & -0.09 & -0.02 & 0.18 & -0.20 & 0.02 & -0.25 & 0.18 & 1.00 \\
\hline
\end{tabular}

\section{Lévy Processes and Return Distributions}

The selection of an appropriate probability distribution model in finance is crucial. Designing the best-fit distribution is the most important step in risk modelling. Black-Scholes theory (Black-Scholes 1973) assumes that asset prices follow Geometric Brownian Motion (GBM). On the other hand, real asset prices such as daily returns show heavy tails, which indicates a behaviour different from the Normal distribution and it leads to kurtosis higher than the Normal distribution. The history of heavy tails in finance began in 1963 with Pareto distribution proposed by Mandelbrot (1963). The proposed model is known as a stable model of asset returns. In finance, modelling asset returns as a heavy-tailed and highly peaked random variable has been well documented. Lévy processes display many appealing properties in financial economics. These models can be thought of as combination of diffusions and jump processes (Chevalier \& Goutte, (2015). In economies, the occurrence of unusual events leads to quite complex dynamics for 
the variable that economist studies. Therefore, the normal behavior of economies is frequently interrupted. This phenomenon uncovers the danger of extreme occurrences. Fat-tailed models have been used enormously in capturing the tail risks of equity markets, optimal portfolio choice, economic hedging, and risk management. In theoretical and applied macroeconomics, researchers have extensively used Gaussian assumptions. But, on the other hand, a huge number of studies have shown fat-tailed models as a better choice than the Gaussian model. The growth rates of macroeconomic variables are seldom normally distributed. Lévy processes in finance are based on more general infinitely divisible distributions comparative to the Normal distribution. These infinitely divisible distributions must be able to represent skewness and excess kurtosis. A distribution is called infinitely divisible if for every positive integer $n$, the characteristic function $\phi(u)$ is also the $n$-th power of a characteristic function. Therefore, for every infinitely divisible distribution a Lévy stochastic process can be defined. Some examples of these frequently used parametric distributions in finance are represented by the generalized hyperbolic distributions (GHD), hyperbolic distribution (HYP), Normal Inverse Gaussian distribution (NIG), variance-gamma distribution(VG). Student's $\mathrm{t}(\mathrm{t})$ and its asymmetric version are also frequently used in financial modelling.

The Generalized Hyperbolic Distribution (GHD) is defined in BarndorffNielsen (1977). The GHD is an infinitely divisible distribution. We can define Lévy processes based on GHD as the stationary process, which starts at zero and has independent increments. The density function of generalized hyperbolic distribution (GHD) is given by:

$f_{X}(x)=a(\lambda, \alpha, \beta, \delta)\left(\delta^{2}+(x-\mu)^{2}\right)^{\frac{\lambda-\frac{1}{2}}{2}} K_{\lambda-1 / 2}\left(\alpha \sqrt{\delta^{2}+(x-\mu)^{2}}\right) e^{\beta(x-\mu)}$

where $a(\lambda, \alpha, \beta, \delta)=\frac{\left(\alpha^{2}-\beta^{2}\right)^{\lambda / 2}}{\sqrt{2 \pi} \alpha^{\lambda-1 / 2} \delta^{\lambda} K_{\lambda}\left(\delta \sqrt{\alpha^{2}-\beta^{2}}\right)}$ and $x, \mu, \lambda \in \mathbb{R}, 0 \leq \delta, 0 \leq|\beta| \leq \alpha$, also $\gamma=\sqrt{\alpha^{2}-\beta^{2}}, K_{v}$ denotes modified third order Bessel function with index $v$. The parameter $\lambda$ defines sub-class of the distribution and $\mu, \delta$ represent scale and location parameters respectively. If $\beta=0$ the distribution is symmetric. For $\lambda=1$, then Hyperbolic distribution (HYP) is given by the density function

$$
f_{X}(x)=\frac{\sqrt{\alpha^{2}-\beta^{2}}}{2 \delta \alpha K_{1}\left(\delta \sqrt{\alpha^{2}-\beta^{2}}\right)} e^{\left(-\alpha \sqrt{\delta^{2}+(x-\mu)^{2}}+\beta(x-\mu)\right)}
$$

where, $x, \mu \in \mathbb{R}, 0 \leq \delta,|\beta| \leq \alpha$.

The NIG distribution in Barndorff-Nielsen (1995) is an infinitely divisible distribution. This class of distributions has semi-heavy tails. The NIG distribution is a special case of the GHD for $\lambda=-1 / 2$. The random variable $X$ follows a NIG distribution if its density function is give $\mathrm{n}$ by:

$$
f_{X}(x)=\frac{\alpha \delta}{\pi} e^{\left(\delta \sqrt{\alpha^{2}-\beta^{2}}+\beta(x-\mu)\right)} \frac{K_{1}\left(\alpha \sqrt{\delta^{2}+(x-\mu)^{2}}\right)}{\sqrt{\delta^{2}+(x-\mu)^{2}}}
$$

where $x, \mu \in \mathbb{R}, 0 \leq \delta, 0 \leq|\beta| \leq \alpha$.

DOI: 10.24818/18423264/54.3.20.03 
The function $K_{1}(x)=\int_{0}^{\infty} \exp \left(-\frac{1}{2} x\left(\tau+\tau^{-1}\right) d \tau\right.$ is a modified Bessel function of third order and index 1 . If we set $\delta=0$ then we obtain another class of the GHD known as VG. This is possible when $\lambda>0$ and $\alpha>|\beta|$.

The class of VG distributions was introduced by Madan and Seneta (1987) as a model for stock returns. The density function of VG is given by:

$$
f_{X}(x)=\frac{\left(\alpha^{2}-\beta^{2}\right)^{\lambda}}{\sqrt{\pi} \Gamma(\lambda)(2 \alpha)^{\lambda-\frac{1}{2}}}|x-\mu|^{\lambda-\frac{1}{2}} K_{\lambda-1 / 2}(\alpha|x-\mu|) e^{\beta(x-\mu)}
$$

The Student's t distribution due to Gosset (1908) is given by:

$$
f_{X}(x)=\frac{K(v)}{\sigma}\left[1+\frac{(x-\mu)^{2}}{\sigma^{2} v}\right]^{-\frac{1+v}{2}}
$$

where $-\infty<x<+\infty,-\infty<\mu<+\infty, \sigma>0, v>0$.

Also, $K(v)=\sqrt{v} B(v / 2,1 / 2)$ and $B$ denotes the beta function given by:

$$
B(a, b)=\int_{0}^{1} t^{a-1}(1-t)^{b-1} d t
$$

Zhu \& Galbraith (2010) introduced the asymmetric student's t distribution. The density function of the distribution is given by:

$$
f_{X}(x)=\frac{1}{\sigma}\left\{\begin{array}{c}
\frac{\alpha}{\alpha^{*}} K\left(v_{1}\right)\left\{1+\frac{1}{v_{1}}\left[\frac{x-\mu}{2 \sigma \alpha^{*}}\right]^{2}\right\}^{-\frac{1+v_{1}}{2}}, \quad \text { if } x \leq \mu \\
\frac{1-\alpha}{1-\alpha^{*}} K\left(v_{2}\right)\left\{1+\frac{1}{v_{2}}\left[\frac{x-\mu}{2 \sigma\left(1-\alpha^{*}\right)}\right]^{2}\right\}^{-\frac{1+v_{2}}{2}}, \quad \text { if } x>\mu
\end{array}\right.
$$

where $-\infty<x<+\infty,-\infty<\mu<+\infty, \sigma>0, v_{1}>0, v_{2}>0,0<\alpha<1$ and

$$
\alpha^{*}=\frac{\alpha K\left(v_{1}\right)}{\alpha K\left(v_{1}\right)+(1-\alpha) K\left(v_{2}\right)}
$$

The maximum likelihood method was used to fit the underlying distributions. The maximum-likelihood estimator is the parameter set that maximizes the likelihood function. The maximum likelihood function is given by:

or the log-likelihood

$$
L(\Theta)=\prod_{i=1}^{n} f_{X}\left(x_{i} ; \Theta\right)
$$

$$
\ln L(\Theta)=\sum_{i=1}^{n} f_{X}\left(x_{i} ; \Theta\right)
$$

where $\left(x_{1}, x_{2}, \ldots, x_{n}\right)$ contains the observed values of the random variable $X$ and $\Theta=\left(\theta_{1}, \theta_{2}, \ldots, \theta_{m}\right)$ are parameters specifying the distribution of random variable. We put $\widehat{\Theta}=\left(\widehat{\theta_{1}}, \widehat{\theta_{2}}, \ldots, \widehat{\theta_{m}}\right)$ and $\widehat{\Theta}$ denote maximum likelihood estimate. In order to choose the best-fit distribution, we consider the Akaike information criterion (AIC), Akaike \& Hirotugu (1974). The AIC is given by:

$$
\mathrm{AIC}=2 m-2 \ln L(\widehat{\Theta})
$$

The smaller the value of criteria better the fitted model. The Akaike information criterion is comprised bias (log likelihood) and variance (parameters) (Hu, 2007), and the larger the log likelihood the better the goodness of fit. 


\section{Fitted Distributions and Risk Modeling}

Financial returns that exhibit heavy-tails are profound to risk managers. It is evident from our data analysis results that; underlying returns have not normally distributed and therefore exhibit fat-tail behavior. We fit various distributions to given financial time series data and, empirical features have been captured with the description of a few parameters. These include at least, the volatility parameter, location parameter, asymmetry measure and finally a parameter describing the decay of the tails. Table 5 presents the parameters estimates of fitted distributions of the Close-return series. Throughout the estimation results, 0.000 should not be interpreted as an absolute zero. We have found evidence of fat-tail distribution for all returns series. Therefore, implemented distributions include asymmetric (symmetric) types of GHD, HYP, NIG, VG, and t. The GHD and asymmetric $\mathrm{t}$ distribution did not converge in some cases.

Table 5. Fitted distributions parameter estimates and diagnostic test for BCH Close-returns

\begin{tabular}{lllllll}
\hline Parameter & lambda & alpha & mu & sigma & gamma & AIC \\
\hline HYP & 1.000 & 0.000 & -0.570 & 6.386 & -0.851 & 1514.47 \\
& $(1.000)$ & $(0.001)$ & $(-0.384)$ & $(6.462)$ & $(0.000)$ & $(1515.75)$ \\
NIG & -0.500 & 0.261 & -0.206 & 7.149 & -0.701 & 1512.26 \\
& $(-0.500)$ & $(0.258)$ & $(-0.465)$ & $(7.231)$ & $(0.000)$ & $(1511.67)$ \\
T & -1.091 & 0.000 & -0.372 & 12.14 & -1.348 & 1516.27 \\
& $(-1.092)$ & $(0.000)$ & $(-0.556)$ & $(12.565)$ & $(0.000)$ & $(1514.96)$ \\
VG & 0.618 & $(0.000)$ & -0.081 & 6.949 & 0.000 & 1506.51 \\
& $(0.553)$ & $(0.000)$ & $(-0.081)$ & $(6.915)$ & $(-0.278)$ & 1507.35 \\
\hline
\end{tabular}

Table 6 displays the parameters estimates of fitted distributions of the Open-return series.

Table 6. Fitted distributions parameter estimates and diagnostic test for $\mathrm{BCH}$ Open-returns

\begin{tabular}{lllllll}
\hline Parameter & lambda & alpha & mu & sigma & gamma & AIC \\
\hline GHD & $(0.516)$ & $(0.012)$ & $(-0.072)$ & $(6.878)$ & $(0.000)$ & $(1492.73)$ \\
HYP & 1.000 & 0.000 & -0.0371 & 6.200 & -0.904 & 1500.94 \\
& $(1.000)$ & $(0.000)$ & $(-0.379)$ & $(6.279)$ & $(0.000)$ & $(1502.34)$ \\
NIG & -0.500 & 0.194 & -0.314 & 7.244 & -0.628 & 1495.51 \\
& $(-0.500)$ & $(0.191)$ & $(-0.495)$ & $(7.334)$ & $(0.000)$ & $(1494.74)$ \\
T & $(-1.000)$ & $(0.000)$ & $(-0.575)$ & $(11.335)$ & $(0.000)$ & $(1499.51)$ \\
VG & 0.542 & $(0.000)$ & -0.006 & 7.059 & -0.694 & $(1487.97)$ \\
& $(0.511)$ & $(0.0000)$ & $(-0.096)$ & $(6.650)$ & $(0.000)$ & $(1428.23)$ \\
\hline
\end{tabular}

DOI: 10.24818/18423264/54.3.20.03 
Muhammad Sheraz, Silvia Dedu

Table 7 presents the parameters estimates of fitted distributions of the Low-return series.

Table 7. Fitted distributions parameter estimates and diagnostic test for $\mathrm{BCH}$ Low-returns

\begin{tabular}{lllllll}
\hline Parameter & lambda & alpha & $\mathrm{mu}$ & sigma & gamma & AIC \\
\hline GHD & $(0.314)$ & $(0.0174)$ & $(0.223)$ & $(7.928)$ & $(0.000)$ & $(1495.92)$ \\
& & & & & & \\
HYP & 1.000 & 0.000 & 0.314 & 6.509 & -1.259 & 1525.60 \\
& $(1.000)$ & $(0.000)$ & $(-0.003)$ & $(6.659)$ & $(0.000)$ & $(1530.01)$ \\
NIG & -0.500 & 0.109 & 0.114 & 8.314 & -1.056 & 1506.51 \\
& $(-0.500)$ & $(0.099)$ & $(-0.064)$ & $(8.722)$ & $(0.000)$ & $(1507.50)$ \\
T & $(-1.000)$ & $(0.000)$ & $(-0.268)$ & $(1329.8)$ & $(0.000)$ & $(1514.44)$ \\
VG & 0.407 & 0.000 & 0.301 & 8.503 & -0.084 & 1491.69 \\
& $(0.468)$ & $(0.000)$ & $(0.301)$ & $(7.581)$ & $(0.000)$ & $(1492.50)$ \\
\hline
\end{tabular}

Table 8 displays the parameters estimates of fitted distributions of the High-return series.

Table 8. Fitted distributions parameter estimates and diagnostic test for $\mathrm{BCH}$ High-returns

\begin{tabular}{lllllll}
\hline Parameter & lambda & alpha & mu & sigma & gamma & AIC \\
\hline GHD & 0.028 & 0.118 & -0.609 & 7.027 & -0.294 & 1462.68 \\
& $(0.006)$ & $(0.122)$ & $(-0.677)$ & $(7.052)$ & $(0.000)$ & $(1461.00)$ \\
HYP & 1.000 & 0.000 & -0.692 & 5.982 & -0.210 & 1481.79 \\
& $(1.000)$ & $(0.000)$ & $(-0.747)$ & $(5.988)$ & $(0.000)$ & $(1480.04)$ \\
NIG & -0.500 & 0.120 & -0.779 & 7.481 & -0.124 & 1464.97 \\
& $(-0.500)$ & $(0.121)$ & $(-0.804)$ & $(7.482)$ & $(0.000)$ & $(1463.03)$ \\
T & -1.000 & 0.000 & -0.893 & 3119.0 & 4.690 & 1474.43 \\
& $(-1.000)$ & $(0.000)$ & $(-0.893)$ & $(1890.61)$ & $(0.000)$ & $(1472.43)$ \\
\hline
\end{tabular}

Based on AIC, the VG distribution best fit Close, Open and Low returns. High returns series followed GHD with a minimum value of AIC. The estimates of symmetric cases for all distribution have given in parenthesis. The results of best fitting distributions show that $\mathrm{BCH}$ (USD) percentage log returns exhibits fat-tail behavior. The Log-Likelihood of $\mathrm{BCH}$ returns fitted distributions are presented in Table 9. 
Bitcoin Cash: Stochastic Models of Fat-Tail Returns and Risk Modeling

Table 9. Log-Likelihood of $\mathrm{BCH}$ returns fitted distributions

\begin{tabular}{llllll}
\hline Dist & T & NIG & HYP & GHD & VG \\
\hline Close & -754.482 & -752.13 & -753.23 & & -749.76 \\
& $(-754.482)$ & $(-752.83)$ & $(-754.87)$ & & $(-750.25)$ \\
Open & & -743.75 & -746.59 & & -739.98 \\
& $(-746.75)$ & $(-744.37)$ & $(-748.17)$ & $(-742.36)$ & $(-741.11)$ \\
Low & -759.98 & -728.48 & -736.89 & -726.34 & -730.41 \\
& $(-733.21)$ & $(-728.51)$ & $(-737.02)$ & $(-726.50)$ & $(-730.92)$ \\
High & & -749.25 & -758.85 & & -741.84 \\
& $(-754.22)$ & $(-750.75)$ & $(-762.60)$ & $(-743.96)$ & $(-743.25)$ \\
\hline
\end{tabular}

\subsection{Q-Q Plots}

The adequacy of the best fitting distributions is assessed in terms of Q-Q plots. The QQ-plot for the best-fitted distribution for each of the four series is given in Fig. 3. For BCH Close-returns, best fitting VG distribution tracks the data very well but not the lower tail. For BCH Open and Low returns, only upper and the lower parts of data have a slightly different track, but in the middle, it captures well. Finally, the $\mathrm{BCH}$ High-returns, best-fitted distribution follows the track of data from uppermiddle to downwards.
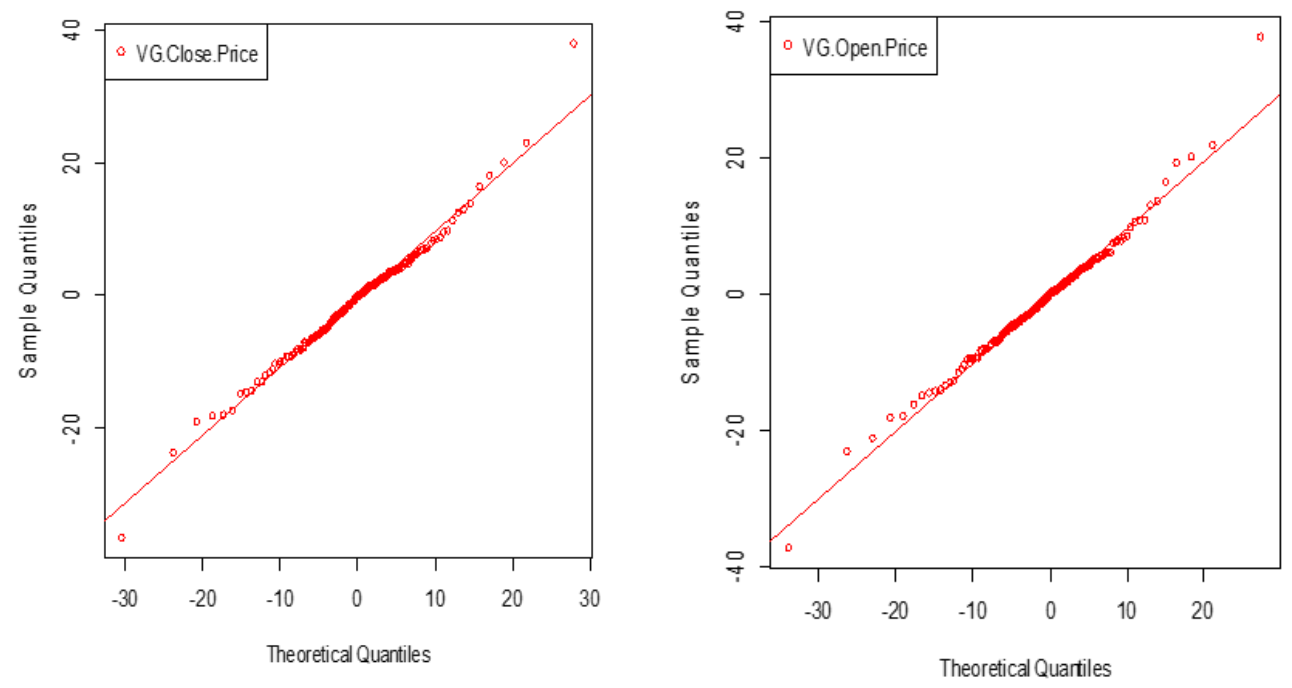

DOI: 10.24818/18423264/54.3.20.03 

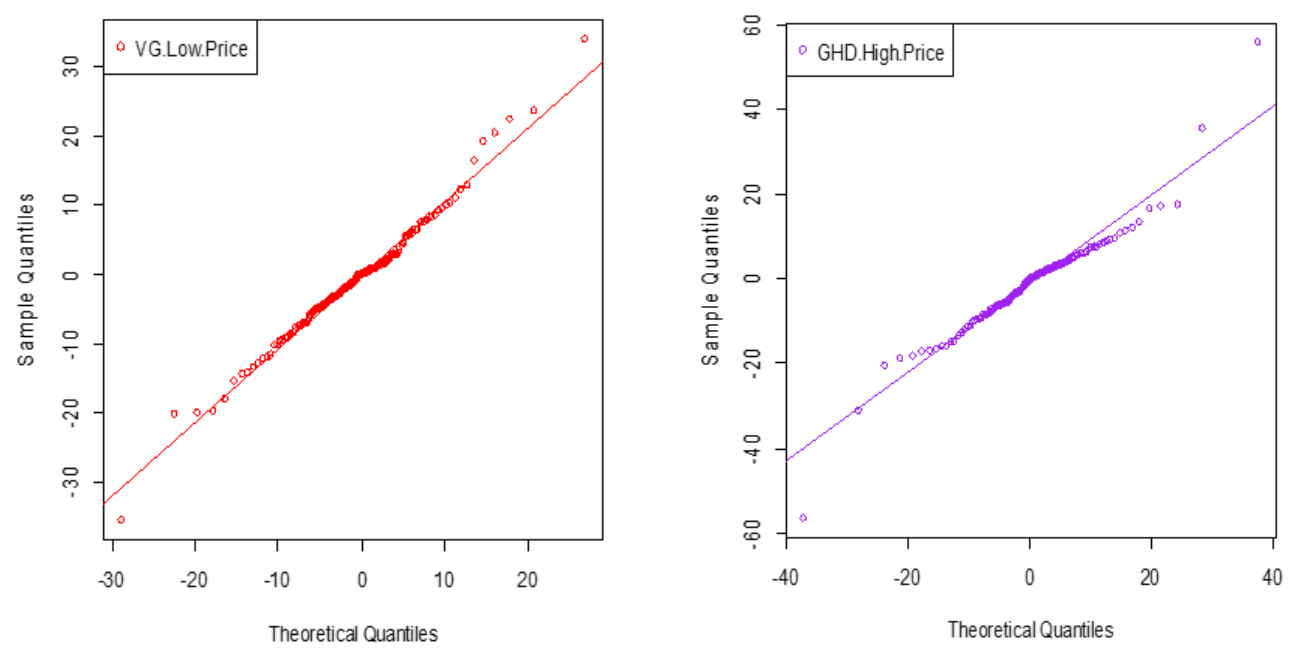

Figure 3. QQ Plot of BCH Close-Open-Low-High Returns

\subsection{Risk Modeling}

In the case of normally distributed financial returns, the standard deviation or volatility of returns captures risk adequately. Financial returns of $\mathrm{BCH}$ exhibit fat-tail distributions. Therefore, it can be misleading. Another, most common risk measure is Value-at-Risk (VaR). It is used to compute losses with respect to market dynamics. VaR has been used frequently in credit risk. Let $\left\{X_{t}\right\}_{t \in T}$ denotes BCH time series process. Suppose $f$ and $F$ denotes marginal density function and distribution function respectively. Then, $\mathrm{VaR}$ is a quantile of distribution function exceeded with probability $1-p$. See details in Jorion (2001) and McNeil et al. (2005). Let $X$ is a random variable then, the Value-at-Risk corresponding to the probability level $\alpha \in[0,1]$ is given by:

$$
\begin{aligned}
& \operatorname{VaR}_{\alpha}(X)=\inf \{x \in \mathbb{R} \mid P(X<-x) \leq 1-\alpha\} \\
& \operatorname{VaR}_{\alpha}(X)=\inf \{x \in \mathbb{R}: F(x) \geq \alpha\}
\end{aligned}
$$

On other hand, if $R_{t}$ denotes log-returns of the underlying time series then, $\mathrm{VaR}_{\alpha}$ is given by:

$$
\mathrm{VaR}_{\alpha}=\mu_{t}+\sigma_{t} \mathrm{q}^{-1}(\alpha)
$$

where $\mathrm{q}$ denotes quantile function of standardized log-returns. Generally, $\alpha$ is taken to be 0.01 or 0.05 . For example, if we say that 1 percent 5 -days VaR is 1 dollar then, it means over the next 5 days, there is a 1 percent chance of losing at least 1 dollar.

Artzner et al. (1999) proposed an alternative risk measure of financial risk referred to as the Expected Shortfall (ES) or tail-VaR. It provides information on the entire tail of the distribution as a single measure. The ES measure is subadditive and allows to compute expected loss when losses exceed VaR. Expected Shortfall estimates the potential size of the loss exceeding $\operatorname{VaR}_{\alpha}(X)$. 
Let $X$ be a random variable. The Expected Shortfall measure corresponding to the random variable $X$ and the probability level $\alpha$ is given by:

$$
\mathrm{E}\left(\mathrm{S}_{\alpha}\right)=\mathrm{E}\left(X \mid X \geq \operatorname{VaR}_{\alpha}(X)\right)
$$

Table 10 and Table 12 display VaR estimates of $\mathrm{BCH}$ closing returns, which are represented in Fig.4. We have compared the results with best-fitted VG against, empirical distribution (EMP), GHD, HYP, NIG, t, and the Normal. We calculate VaR over the period, from the $95 \%$ to $99.9 \%$ probability level. Therefore, probability of losses exceeding VaR is given by $p=(0.001,0.05,0.001)$. VaR is a quantile on the underlying return distributions for $\mathrm{BCH}$ data. It is evident from Fig 4 that VG and HYP track the empirical level quite good. Table 10 provides estimation results ranging from $0.1 \%, 1 \%, 2 \%, 3 \%, 4 \%$ and $5 \%$.

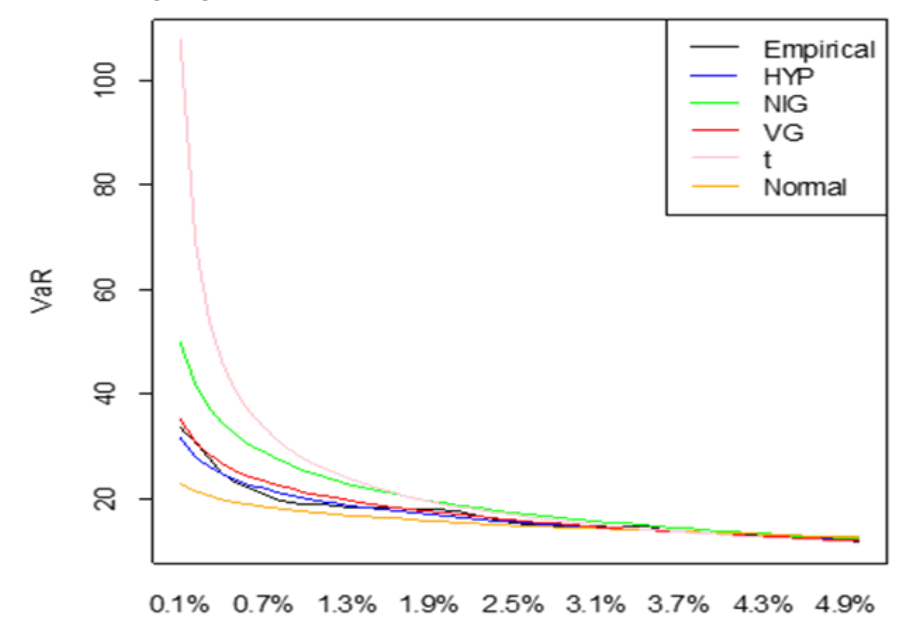

Figure 4. VaR estimates of $\mathrm{BCH}$ Close price returns

The risk is overestimated by NIG and t models, while normal distribution underestimates the risk. For the case of $\mathrm{BCH}$ Low-returns, we provide VaR computations on best-fitted VG and comparison with the empirical and normal distribution (see Table 10).

Table 10. Value-at-Risk estimates of $\mathrm{BCH}$ (USD) closing price return series

\begin{tabular}{lllllll}
\hline \multicolumn{1}{c}{$\boldsymbol{\alpha}$} & $0.1 \%$ & $1 \%$ & $2 \%$ & $3 \%$ & $4 \%$ & $5 \%$ \\
\hline EMP & 33.531 & 18.832 & 17.538 & 14.597 & 13.114 & 11.729 \\
VG & 34.959 & 21.106 & 17.030 & 14.678 & 13.026 & 11.757 \\
HYP & 31.340 & 19.914 & 16.475 & 14.463 & 13.035 & 11.928 \\
NIG & 49.734 & 25.159 & 19.019 & 15.775 & 13.642 & 12.088 \\
t & 107.591 & 27.392 & 18.840 & 15.140 & 12.936 & 11.423 \\
NOR & 22.767 & 17.364 & 15.435 & 14.212 & 13.292 & 12.543 \\
\hline
\end{tabular}

DOI: 10.24818/18423264/54.3.20.03 
Muhammad Sheraz, Silvia Dedu

The Expected Shortfall measure is computed for the best-fitted VG against empirical, NIG, HYP, $\mathrm{t}$ and normal distribution. The normal distribution and HYP underestimate the ES, whereas $t$ and NIG overestimates it. Only the VG model follows the empirical track, as results from Table 11.

Table 11. Expected Shortfall estimates of $\mathrm{BCH}$ closing price return series

\begin{tabular}{lllllll}
\hline \multicolumn{1}{c}{$\boldsymbol{\alpha}$} & $0.1 \%$ & $1 \%$ & $2 \%$ & $3 \%$ & $4 \%$ & $5 \%$ \\
\hline EMP & 36.511 & 26.467 & 23.149 & 20.314 & 19.015 & 17.931 \\
VG & 41.089 & 27.106 & 22.968 & 20.569 & 18.880 & 17.577 \\
HYP & 36.302 & 24.876 & 21.437 & 19.425 & 17.997 & 16.890 \\
NIG & 62.119 & 35.614 & 28.646 & 24.853 & 22.301 & 20.406 \\
t & 829.355 & 123.019 & 72.685 & 54.057 & 44.033 & 37.654 \\
NOR & 24.725 & 19.761 & 18.032 & 16.951 & 16.147 & 15.499 \\
\hline
\end{tabular}

Value-at-Risk estimates for $\mathrm{BCH}$ low prices returns are presented in Table 12.

Table 12. VaR estimates of $\mathrm{BCH}$ low price return series

\begin{tabular}{lllllll}
\hline \multicolumn{1}{c}{$\boldsymbol{\alpha}$} & $0.1 \%$ & $1 \%$ & $2 \%$ & $3 \%$ & $4 \%$ & $5 \%$ \\
\hline EMP & 31.935 & 20.009 & 16.254 & 14.327 & 12.672 & 11.846 \\
VG & 33.134 & 20.018 & 16.177 & 13.967 & 12.418 & 11.231 \\
NOR & 22.223 & 16.953 & 15.073 & 13.880 & 12.982 & 12.252 \\
\hline
\end{tabular}

Expected Shortfall estimates for BCH low prices returns are displayed in Table 13. Again, the VG model estimates well as compare to others.

Table 13. ES estimates of BCH low price return series

\begin{tabular}{lllllll}
\hline \multicolumn{1}{c}{$\boldsymbol{\alpha}$} & $0.1 \%$ & $1 \%$ & $2 \%$ & $3 \%$ & $4 \%$ & $5 \%$ \\
\hline EMP & 35.527 & 25.275 & 22.742 & 19.738 & 18.429 & 17.396 \\
VG & 38.959 & 25.696 & 21.784 & 19.521 & 17.929 & 16.704 \\
NOR & 24.134 & 19.291 & 17.606 & 16.551 & 15.767 & 15.135 \\
\hline
\end{tabular}

\section{Conclusions}

This paper provides a statistical analysis of several stochastic models of fat-tail returns and risk models. The evidence of fat-tailed returns distribution for the $\mathrm{BCH}$ data is investigated, by performing a statistical analysis of Bitcoin Cash $(\mathrm{BCH})$ in the U.S. dollar. The results obtained by analyzing daily Close, Open, Low, and High returns of $\mathrm{BCH}$ data series show that the returns follow the generalized hyperbolic distribution and variance gamma distribution.

Recently, the use of heavy-tail distribution in risk management and financial time series has got significant attention in the financial literature. The research on famous cryptocurrency Bitcoin (BTC) suggests that the generalized 
hyperbolic distribution gives the best fit (see Chen et al., 2017). We have analyzed the Bitcoin Cash returns instead of the Bitcoin. The results obtained in this paper prove that variance-gamma distribution best fit the close, open and low returns, where high returns follow the generalized hyperbolic. In some cases, a small difference in AIC has been found. For the entire period (Jun 2018 to Jan 2019) peaks of the returns, distribution has found much higher and tails are fatter than the Normal distribution. Therefore, there are more trading days when underlying prices have not been predicted using Normal distribution. Open and High return series for whole period shows high correlation. On the other hand, monthly-divided daily returns follow the high peak distribution in a few cases. BCH returns in September and December are highly correlated. The three months, November and December in 2018, respectively January 2019, have been found to be the most volatile. The monthly-divided daily returns of Open, Close, Low and High returns follow quite the same volatility pattern except for Jan 2019, when Low-return series has almost $16 \%$ volatility. Therefore, if we can capture predictability in volatility, it may be possible to improve portfolio decisions, risk management option pricing, among other applications. Empirical facts from our study explore high volatility in the exchange rate of BTC to USD. Therefore, investors have faced severe market risk. Besides volatility, computations performed using two other risk measures, respectively Value-at-Risk and Expected Shortfall, show the VG model follows the empirical track.

\section{REFERENCES}

[1] Akaike, H. (1974), A New Look at the Statistical Model Identification. IEEE Transactions on Automatic Control, 19:716-23;

[2] Artzner, P., Delbaen, F., Eber, J.M., Heath, D. (1997), Thinking Coherently. Risk, 10: 68-71;

[3] Artzner, P., Delbaen, F., Eber, J.M., Heath, D. (1999), Coherent Measures of Risk. Mathematical Finance, 9:203-228;

[4] Bariviera, A.F., Basgall, M.J., Hasperué, W., Naiouf, M. (2017), Some

Stylized Facts of the Bitcoin Market. Physica A: Statistical Mechanics and Applications, 484:82-90;

[5] Barndorff-Nielsen, O. (1977), Exponentially Decreasing Distributions for the Logarithm of Particle Size; Proceedings of the Royal Society of London. Series A, 353:401- 409;

[6] Black, F., Scholes, M. (1973), The Pricing of Options and Corporate

Liabilities; Journal of political economics, 81(3):677-654;

[7] CoinMarketCap (2019), Cryptocurrency Market Capitalizations;

https://coinmarketcap.com;

[8] Corlu, C.G., Corlu, A. (2015), Modelling Exchange Rate Returns: Which

Flexible Distribution to Use? Quantitative Finance, 15:1851-64;

DOI: 10.24818/18423264/54.3.20.03 
[9] Dyhrberg, A.H. (2016), Bitcoin, Gold and the Dollar - A GARCH Volatility Analysis. Financial Research Letters, 16:85-92;

[10] Eberlein E., Keller, U. (1995), Hyperbolic Distributions in Finance. Bernoulli,1:281-299;

[11] Gosset, W.S. (1908), The Probable Error of a Mean. Biometrika, 6:1-25;

[12] Chu, J., Nadarajah, S., Chen, S. (2015), Statistical Analysis of the

Exchange Rate of Bitcoin. PLOS ONE, 10(7);

[13] Jorion, P. (2001), Value at Risk: The New Benchmark for Measuring

Financial Risk. 2nd edn. McGraw-Hill, New York;

[14] Linden, M. (2001), A Model for Stock Return Distribution. International

Journal of Finance \& Economics, 6:159-69;

[15] Mandelbrot, B. (1963), The Variation of Certain Speculative Prices. Journal of Business, 36:394-419;

[16] McNeil, A., Frey, R., Embrechts, P. (2005), Quantitative Risk

Management: Concepts, Techniques and Tools. Princeton University Press, Princeton, NJ;

[17] Osterrieder, J., Lorenz, J. (2017), A Statistical Assessment of Bitcoin and

Its Extreme Tail Behavior. Annals of Financial Economics, 12 (1);

[18] Rama, C. (2001), Empirical Properties of Asset Return: Stylized Facts and

Statistical Issues. Quantitatice Finance, 1:23-236;

[19] RiskMetrics Group (1994), RiskMetrics Technical Document, Technical

Report. J.P. Morgan, NewYork;

[20] Chan, S., Chu, J., Nadarajah, S., Osterrieder, J. (2017), A Statistical

Analysis of Cryptocurrencies. Journal of Risk and Financial Management, 10, 12;

[21] Schoutens, W. (2003), Lévy Processes in Finance: Pricing Financial

Derivatives. John Wiley \& Sons, Ltd.;

[22] Rachev, S.T. (2003), Handbook of Heavy Tailed Distribution in Finance.

Elsevier, The Netherlands;

[23] Zaven, A.K., Dudewicz, E.J., Shimizu, C. (2010), Handbook of Fitting

Statistical Distributions with R. CRC press Taylor \& Francis Group. 